\title{
Stroke-like manifestations in a patient with Listeria monocytogenes abscess and Horton's arteritis
}

\author{
Dimitri Peterlana, ${ }^{1}$ Susanna Cozzio, ${ }^{1}$ Domenico Marco Bonifatti, ${ }^{2}$ Walter Spagnolli ${ }^{1}$ \\ ${ }^{1}$ Internal Medicine Unit, S. Chiara Hospital; ${ }^{2}$ Stroke Unit, Department of Neurosciences, S. Chiara Hospital, APSS, Trento, Italy
}

\begin{abstract}
Brain abscesses due to Listeria monocytogenes are rare and are a clinical challenge. We report the case of a 72-year old female patient under immunosuppressive treatment for Horton's arteritis who was admitted with an initial diagnosis of ischemic stroke. Further workup revealed listerial abscesses as the cause of her neurological symptoms. She was treated with ampicillin plus gentamicin with an excellent outcome. In spite of the rarity of this entity, immunosuppresive drugs are the most important predisposing factors. These drugs are widely used and consequently there is an increased number of subjects at risk of listerial brain abscesses.
\end{abstract}

\section{Introduction}

Listeria monocytogenes is an uncommon cause of illness in the general population. However, it can cause serious life-threatening infections especially in neonates and pregnant women. Other groups of patients at increased risk of listeriosis are those at the extremes of age and those who are immunosuppressed, specifically those with defects in cell-mediated immunity, such as transplanted subjects, patients receiving immunosuppressive therapy for autoimmune diseases,

Correspondence: Dimitri Peterlana, Medicina Interna, Ospedale S. Chiara, P.le Largo Medaglie d'Oro 10, 38100 Trento, Italy.

Tel.: +39.0461.903678

E-mail: dimitri.peterlana@apss.tn.it

Key words: stroke, Listeria monocytogenes, cerebral abscess, immunosuppression.

Contributions: DP and SC wrote the manuscript; DMB and WS reviewed the manuscript.

Conflict of interests: the authors declare that they have no conflict of interests.

Conference presentation: the case was presented at the XVI FADOI National Congress in Florence, 15-18 May, 2011.

Received for publication: 8 May 2013.

Revision received: 8 May 2013

Accepted for publication: 27 June 2013.

This work is licensed under a Creative Commons Attribution NonCommercial 3.0 License (CC BY-NC 3.0).

CCopyright D. Peterlana et al., 2014

Licensee PAGEPress, Italy

Italian Journal of Medicine 2014; 8:64-68

doi:10.4081/itjm.2013.384 malignancies and AIDS. ${ }^{1}$ The most common manifestation of listerial infection involving the central nervous system (CNS) is meningoencephalitis; other less common manifestations include rhomboencephalitis, i.e. brainstem encephalitis (encephalitis of the pons and medulla), and cerebritis with abscess in the absence of meningitis. ${ }^{2,3}$ Several reviews and reports of listerial CNS infection have been published, but only 42 cases of brain abscesses have been reported in the English literature so far. ${ }^{4-6}$ Here we describe a new case of brain abscesses due to Listeria monocytogenes in a patient with Horton's arteritis receiving immunosuppressive therapy who presented with left hemiplegia of sudden onset.

\section{Case Report}

A 72-year old Italian woman was admitted to the Internal Medicine ward of Trento Hospital because of left hemiplegia that had begun suddenly a few hours earlier. Her relatives reported no others symptoms at home, such as fever, headache or seizures. She had been diagnosed as having Horton's arteritis 3 months earlier and was being treated with methylprednisone $(35 \mathrm{mg} /$ day), methotrexate $(7.5 \mathrm{mg} /$ week $)$ and aspirin $(100 \mathrm{mg} /$ day $)$. She was also known to have type 2 diabetes mellitus and arterial hypertension. On admission, neurological examination showed conserved consciousness, left hemiplegia with ipsilateral hypoesthesia, extensor plantar reflex on the left and no deficit involving the cranial nerves. The physical examination was otherwise normal; the patient had no fever, her blood pressure was $170 / 86 \mathrm{mmHg}$, and she had a regular heart rate of 90 beats per minute. An initial laboratory workup showed a normal white blood cell count $\left(9.4 \times 10^{9} / \mathrm{L}: 68 \%\right.$ neutrophils, $27.8 \%$ lymphocytes), hemoglobin concentration $(13.5 \mathrm{~g} / \mathrm{dL})$, and C-reactive protein level $(5.24$ $\mathrm{mg} / \mathrm{L}$; normal value $<6 \mathrm{mg} / \mathrm{L}$ ). Routine biochemistry 
and chest radiography were normal and serial electrocardiograms revealed sinus rhythm. A brain computed tomography (CT) scan in non-contrast mode showed a right-sided hypodense lesion involving the basal ganglia and posterior limb of the internal capsule; another hypodense lesion was present in the posterior part of the ipsilateral side of the pons (Figure 1), compatible with a recent infarction. Two days later the patient developed a single episode of fever $\left(38.8^{\circ} \mathrm{C}\right)$ together with mild lethargy but no worsening of her motor deficit and without any other signs or symptoms. In accordance with our general policy, we performed blood and urine cultures and repeated the blood analysis which showed only minimal increases of C-reactive protein level (11.4 $\mathrm{mg} / \mathrm{L})$ and white blood cell count $\left(11.8 \times 10^{9} / \mathrm{L}\right)$ with a normal pro-calcitonin concentration $(0.16 \mathrm{ng} / \mathrm{mL}$; normal value $<0.5 \mathrm{ng} / \mathrm{mL}$ ). Blood culture results were available $24 \mathrm{~h}$ later and were positive for Listeria monocytogenes. Intravenous treatment with ampicillin 12 $\mathrm{g} /$ day and gentamicin $240 \mathrm{mg} /$ day was started immediately. A brain contrast $\mathrm{CT}$ scan and magnetic resonance imaging (MRI) (without gadolinium) demonstrated two lesions with ring-like enhancement located in the posterior limb of the internal capsule and pons, compatible with listerial abscesses (Figure 2). The antibiotic therapy was continued for 10 weeks. Neuroimaging followup at 3 weeks and 3 months (Figure 3) showed considerable improvement. The clinical course was favorable after a long period of neurological rehabilitation, and the residual deficit was minimal.

\section{Discussion}

Listeria monocytogenes is a Gram-positive, catalasepositive facultative anaerobic rod. Listeria can be isolated from soil, food, animal feed, water and domestic animals as well as from asymptomatic humans. ${ }^{7}$ Studies have shown that outbreaks and sporadic episodes of listeriosis are principally food-borne, typically through dairy products. However, it is often difficult to identify the onset of the infection, especially in sporadic cases, considering that the incubation time is 30 days from ingestion to presentation. ${ }^{8,9}$ Listeria typically enters the body through the gastrointestinal tract. Once in the gut, Listeria can invade the intestinal epithelium without disrupting the integrity of the intestinal wall. The portal circulation then carries the pathogen to the liver and from there to the blood. Many tissues may be then infected with a focal infectious syndrome, with the most notable and frequent being the CNS. The immune response of the host is important in determining the course of the infection. The innate immune response plays a vital role in limiting and protecting the host during the initial phases of infection but the acquired immune response, mainly cell-mediated, is required to eliminate the infection. ${ }^{10}$ The clinical manifestations of Listeria infection range from a febrile gastroenteritis that is self-limited to an invasive listeriosis. ${ }^{7}$ Apart from maternal-fetal and neonatal listeriosis, not discussed here, the most fre-
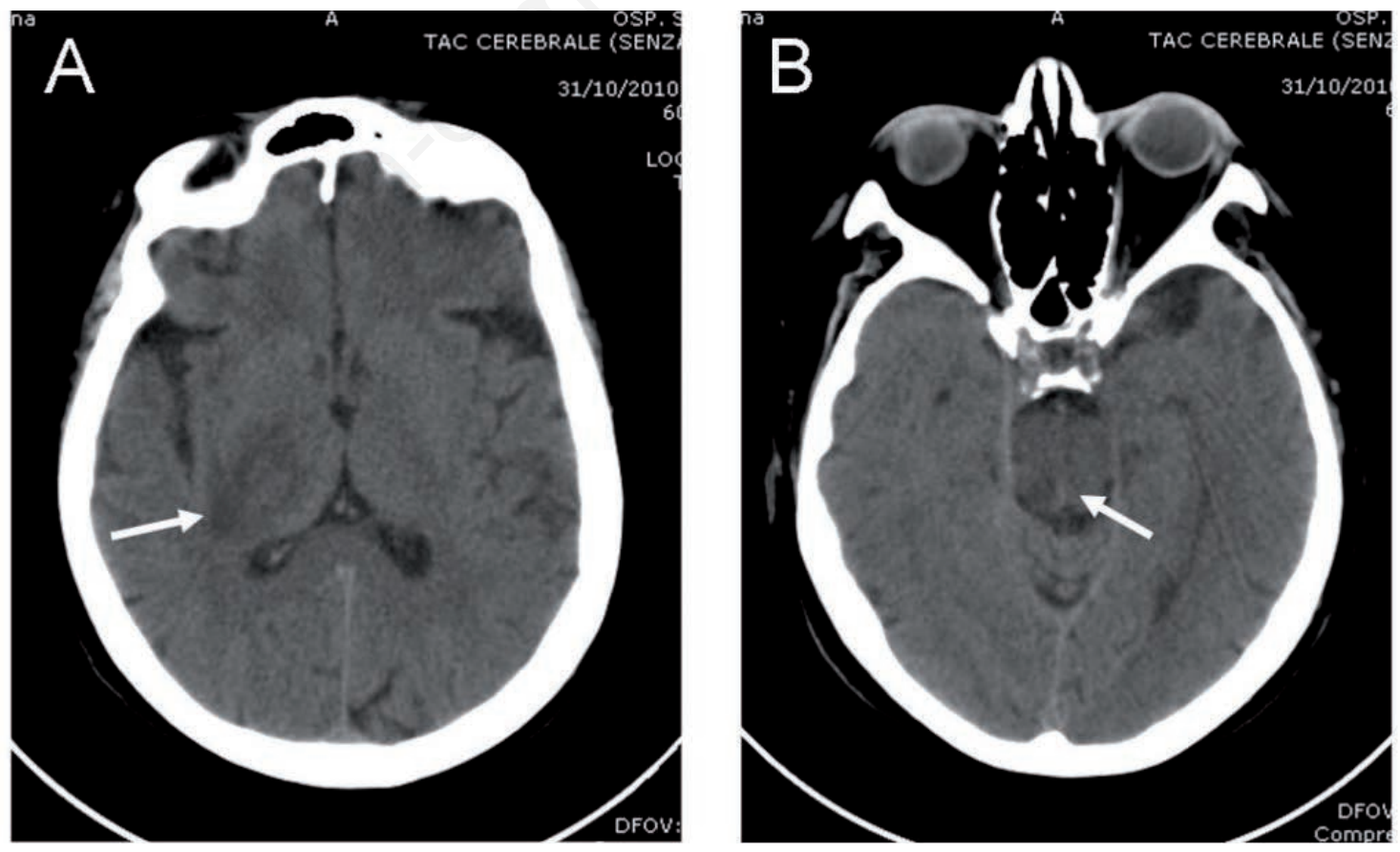

Figure 1. Non-enhanced axial brain computed tomography scan on admission showing hypodense lesions in the basal ganglia and posterior limb of the internal capsule (arrow) (A); analogous lesion in the posterior part of the ipsilateral pons (arrow) (B). 
quent form of invasive infection in adults is a bacteremic state followed by meningitis. Listeria is one of the four major pathogens responsible for communityacquired bacterial meningitis. ${ }^{4}$ In addition to meningitis and meningoencephalitis, less commonly listerial CNS infection may present as brainstem encephalitis and brain abscesses. ${ }^{1-3}$ To our knowledge, only 42 cases of Listeria brain abscesses have been published so far, with the majority presenting as solitary abscesses. In contrast to patients with rhomboencephalitis, who are otherwise healthy, brain abscesses are typically found in subjects with an underlying illness or immunosuppressed state. ${ }^{4}$ Most of the cases reported have occurred in renal transplant recipients or patients affected by leukemia, AIDS, or other immunological disorders including Crohn's disease, autoimmune hepatitis and primary biliary cirrhosis who were being treated with steroids and immune-suppressive agents. ${ }^{4-6}$

The patient described here was immunodepressed, with a relatively short history of steroid and methotrexate therapy for Horton's arteritis. She also suffered from diabetes, even if this was well-controlled with dietary measures. She was admitted for signs and symptoms that resembled those of an ischemic stroke. The initial brain CT scan showed a hypodense lesion in the left internal capsule still compatible with an ischemic lesion without clear edema or a mass effect, making our patient's presentation even more subtle. Moreover she had no fever or meningeal signs and acute phase reactants were only slightly above the normal range. Typical neuroimaging signs were present a few days later when contrast CT and brain MRI showed the classical ring enhanced lesion with massive edema. She had positive blood cultures as do the majority, if not all, of the patients with abscesses described in the literature. ${ }^{4}$ It has been suggested that, as a consequence of the immunological status, the frequency of Listeria bacteremia is higher
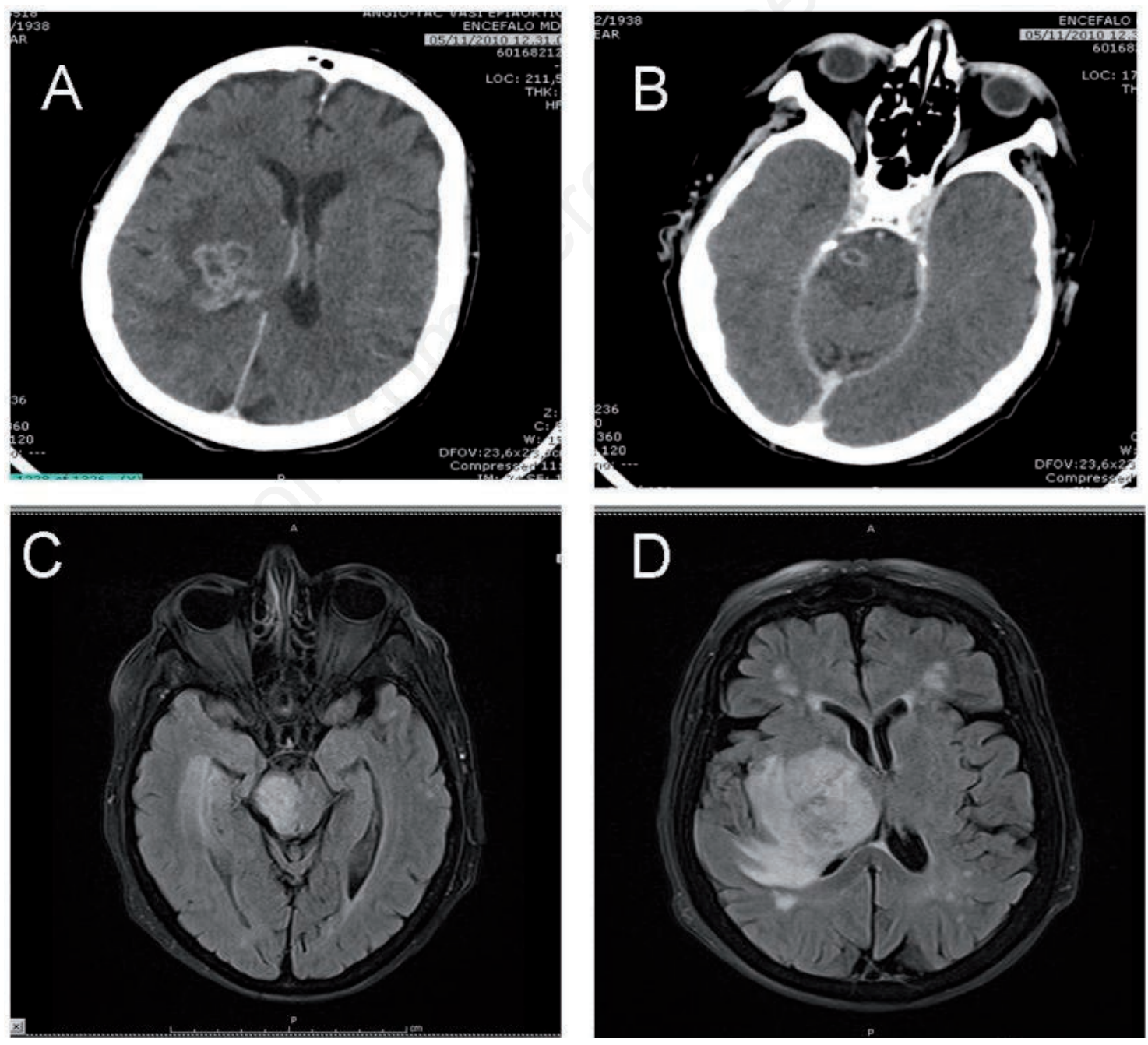

Figure 2. Contrast computed tomography $(A, B)$ and magnetic resonance imaging $(C, D)$ of the brain demonstrating ring-enhancing lesions and surrounding edema in posterior arm of the internal capsule and in pons. 
in patients with abscesses, while the rate of positive cultures is lower in cases of meningitis $(75 \%$, mostly in immunocompromised subjects) and rhomboencephalitis (61\%, mostly in healthy subjects). ${ }^{11}$ We did not examine the cerebrospinal fluid because the patient had no meningeal signs and because we considered the positive blood culture and typical neuroimaging signs sufficient for the diagnosis. Culture of the cerebrospinal fluid may be helpful, but it is positive in less that half of the cases with brain abscesses. ${ }^{4,11}$ Imaging studies performed with CT and MRI are particularly helpful in demonstrating the abscesses and confirming the diagnosis. ${ }^{12}$ Neuroimaging is also essential for monitoring the response to treatment and during follow-up.

Our patient was treated with ampicillin in association with gentamicin, which is the treatment of choice suggested by most authorities for the treatment of listerial bacteremia and CNS involvement, especially in pa- tients with impaired T-cell function. ${ }^{13}$ For subjects allergic to penicillins, trimethoprim/sulfamethoxazole may be used as an alternative. Due to the intracellular location of Listeria and its difficult clearance from brain tissue, the treatment of listerial abscesses is necessarily long, requiting up to 6 weeks, in order to minimize the risk of relapses or recurrences. In our patient antibiotic therapy was continued for 10 weeks, with considerable clinical and radiological improvements.

\section{Conclusions}

In conclusion, we report a clinical case of Listeria brain abscess in a patient with impaired immunity due to immunosuppressive therapy with methotrexate and steroids. The patient's clinical presentation was initially suggestive of ischemic stroke but the following workup revealed a different cause of her symptoms.
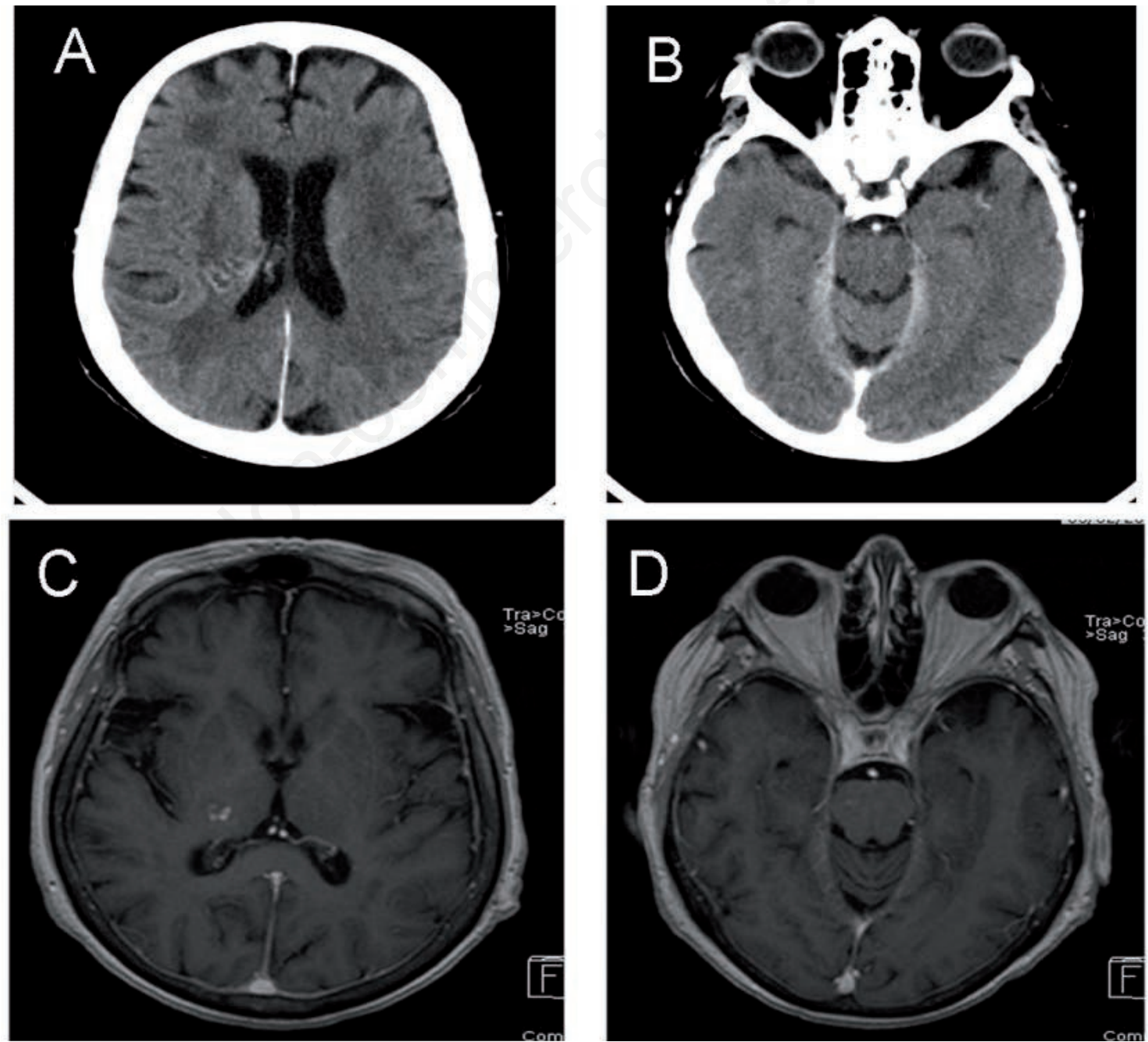

Figure 3. Neuro-imaging performed at 3 weeks $(A, B)$ and at 3 months $(C, D)$ after treatment showing a marked reduction of the enhanced lesions and complete resolution of edema. 


\section{References}

1. Mylonakis E, Hohmann EL, Calderwood SB. Central nervous infection with Listeria monocytogenes. 33 years experience at a general hospital and review of 776 episodes from the literature. Medicine (Baltimore) 1998;77:313-36.

2. Reynaud L, Graf M, Gentile I, et al. A rare case of brainstem encephalitis by Listeria monocytogenes with isolated mesencephalic localization. Case report and review. Diagn Microbiol Infect Dis 2007;58:121-3.

3. Cone LA, Leung MM, Byrd GR, et al. Multiple cerebral abscesses because of Listeria monocytogenes: three case reports and a literature review of supratentorial listeria brain abscess(es). Surg Neurol 2003;59:320-8.

4. Bartt R. Listeria and atypical presentations of Listeria in central nervous system. Semin Neurol 2000;20:361-73.

5. Gamaletsou M, Sipsas NV, Zormpala A, et al. A suspicious stroke. Am J Med 2008;11:858-61.

6. Al-Khatti AA, Al-Tawfiq JA. Listeria monocytogenes brain abscess in a patient with multiple myeloma. J Infect Dev Ctries 2010;4:849-51.

7. Lober B. Listeriosis. Clin Infect Dis 1997;24:1-11.

8. McLauchlin J. Human listeriosis in Britain, 1967-85: a summary of 722 cases. Epidemiol Infect 1990;104: 191-201.

9. Drevets DA, Bronze MS. Listeria monocytogenes: epidemiology, human disease, and mechanisms of brain invasion. FEMS Immunol Med Microbiol 2008;53: 151-65.

10. Cossart P. Listeriology (1926-2007): the rise of a model pathogen. Microbes Infect 2007;9:1143-6.

11. Dee RR, Lorber B. Brain abscess due to Listeria monocytogenes: case report and literature review. Rev Infect Dis 1986;8:968-77.

12. Aladro Y, Ponce P, Angel-Moreno A, Santana MA. Cerebritis due to Listeria monocytogenes: $\mathrm{CT}$ and MR findings. Eur Radiol 1996;6:188-91.

13. Lorber B. Treatment of brain abscess due to Listeria monocytogenes. Clin Infect Dis 2005;41:419. 